\title{
ESTUDIO DEL ESTÁNDAR WATERML
}

\author{
BETSY MARIBY SALAS CHALLCO ${ }^{1}$, JOAN MASÓ PAU ${ }^{2}$, LLUÍS PESQUER \\ MAYOS $^{2}$ \\ ${ }^{1}$ Universitat Autònoma de Barcelona. \\ 08193 Bellaterra, España \\ betmarys@gmail.com \\ ${ }^{2}$ Grumets research group. CREAF. Campus de la Universitat Autònoma de Barcelona. \\ 08193 Bellaterra, España \\ 1.pesquer@creaf.uab.cat, joan.maso@uab.cat
}

\begin{abstract}
RESUMEN
Los estándares para la información geoespacial distribuida pueden ser de propósito general o particulares de un dominio. Algunos dominios han avanzado más que otros en la estandarización y en la interoperabilidad como por ejemplo el dominio MeteorológicoClimático o el Hidrológico. El colectivo de Hidrología detectó la necesidad del estándar WaterML, el cual ayuda a la óptima gestión de los recursos del agua. En términos generales, WaterML es un estándar que define un modelo de datos para la representación de las observaciones hidrológicas, con la intención de permitir el intercambio de este conjunto de datos a través de sistemas de información. Está basado en dos estándares: Geographic Markup Language (GML) y Observations and Measurements (O\&M). WaterML en su versión 2.0 fue desarrollado por Open Geospatial Consortium (OGC), fue aprobado a mediados del 2012 y finalmente publicado a inicios del 2014. A pesar de ser un estándar emergente, diversas instituciones gubernamentales y no gubernamentales han considerado útil estandarizar la información sobre sus series temporales de datos hidrológicos y además publicarlas en la web. Este trabajo revisa y cuantifica el nivel de uso de WaterML en las aplicaciones hidrológicas actuales y sitúa a WaterML en la arquitectura del conjunto de los estándares OGC.
\end{abstract}

Palabras clave: agua, hidrología, estándar, web, codificación.

\section{STUDY OF THE WATERML STANDARD}

\section{ABSTRACT}

Standards for distributed geospatial information can be general purpose or specific of a domain. Some domains have advanced more than others in the standardization and interoperability such as the Weather-Meteorological domain or the Hydrological. The Hydrology community detected the need of the WaterML standard, which helps with the 


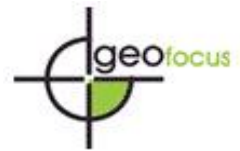

Salas Challco B. M., Masó Pau, J. y Pesquer Mayos, L. (2017): "Estudio del estándar WaterML", GeoFocus (Artículos), $n^{\circ}$ 19, p. 129-150. ISSN: 1578-5157 http://dx.doi.org/10.21138/GF.485

optimal management of water resources. In general terms, WaterML is a standard that defines a data model for the representation of hydrologic observations, with the intention of allowing the exchange of this data through information systems, based on two standards: Geographic Markup Language (GML) and Observations and Measurements (O\&M). WaterML version 2.0 was developed by the Open Geospatial Consortium (OGC); it was approved in mid-2012 and finally published at the beginning of 2014. Despite being an emerging standard, various governmental and non-governmental institutions have been considered useful to standardize the information about its time series of hydrological data and also share them on the web. This work reviews and quantifies the level of use of WaterML in current hydrological applications and places WaterML in the architecture within the OGC set of standards.

Keywords: water, hydrology, standard, web, coding, encoding.

\section{Introducción:}

El agua dulce es uno de los recursos naturales más preciados por el hombre. La Organización Meteorológica Mundial define que el agua es esencial para la vida, para generar energía hidroeléctrica y para satisfacer las necesidades de riego y de los hogares. Para garantizar la gestión y el suministro de este bien, debemos proteger los sistemas de agua vulnerables, mitigar los impactos de los peligros relacionados con el agua, salvaguardar el acceso a las funciones y servicios de agua y administrar los recursos hídricos de manera integrada y equitativa (UNESCO, 2015). En este propósito existen diferentes organizaciones gubernamentales y no gubernamentales que han puesto énfasis en contar con tecnologías para administrar los recursos hídricos, protegerlos contra la contaminación, y tomar medidas preventivas frente a los diferentes fenómenos naturales como las inundaciones o sequías (Castaño, Gómez-Alday y Sanz, 2008; Diez, 1995).

La Hidrología es la ciencia que estudia las aguas terrestres su origen, su movimiento y su distribución en nuestro planeta, sus propiedades físicas y químicas, su interacción con el medio ambiente físico y biológico y su influencia sobre las actividades humanas (Llamas, 1993; Burillo y Sanz, 1960).

Teniendo en cuenta la amplitud de esta ciencia y el número considerable de temas, disciplinas y materias que abarca, se han desarrollado técnicas hidrológicas en el marco de la gestión de los recursos hídricos tales como por ejemplo: diseño de redes; métodos de observación; transmisión de datos; recopilación; proceso y almacenamiento de datos; elaboración de modelos; teledetección; análisis hidrológico para la planificación y el diseño de estructuras de ingeniería y sistemas de recursos hídricos; formulación de metodologías, técnicas y mejores prácticas para la predicción hidrológica y la gestión de recursos hídricos; entre otros. Por otra parte, para complementar estás técnicas están las herramientas y tecnologías hidrológicas como son por ejemplo: programas informáticos y/o aplicaciones web; sistemas de información geográfica (SIG); instrumentos de planificación hidrográfica y ambiental; modelación de sistemas de recursos hídricos; estándares aplicados al sector del agua, entre otros (World Meteorological Organization, 2015).

En este contexto, el Hidrology Domain Working Group (DWG) es un grupo de trabajo conjunto entre la World Meteorological Organization (WMO) y el Open Geospatial Consortium (OGC) (Lefort, 2009) que ha desarrollado el estándar WaterML en su versión 2.0 


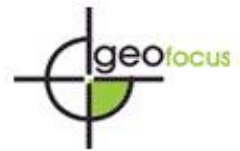

Salas Challco B. M., Masó Pau, J. y Pesquer Mayos, L. (2017): "Estudio del estándar WaterML", GeoFocus (Artículos), $n^{\circ}$ 19, p. 129-150. ISSN: 1578-5157 http://dx.doi.org/10.21138/GF.485

para el intercambio de datos hidrológicos. WaterML 2.0 se ha iniciado y diseñado por un grupo de organizaciones nacionales e internacionales tales como Commonwealth Scientific and Industrial Research Organization (CSIRO), Consortium of Universities for Advancement of Hydrologic Science Incorporated (CUAHSI), United States Geological Survey (USGS), Bureau of Meteorology (BOM), National Oceanic and Atmospheric Administration (NOAA), KISTERS, entre otros, los cuales comparten sus progresos (Khattar y Ames, 2014).

El objetivo del presente trabajo es realizar el estudio de las principales características del estándar y demostrar con una recopilación de ejemplos como se ha venido aplicando el estándar WaterML desde su versión 1.0 (1.1) de CUAHSI hasta su versión 2.0 de OGC.

Por consiguiente, WaterML2.0 es un estándar abierto para la codificación de datos de observaciones de agua, se basa en Observations \& Measurements 2.0 (O\&M, ISO19156) y es implementado como un esquema de aplicación de acuerdo a las reglas de Geography Markup Language 3.2 (GML). En este propósito, explica como codificar la información hidrológica en Extensible Markup Language (XML) y actuar como formato de intercambio vía internet a través de servicios web (Giuliani et al, 2013).

XML es un formato que permite la lectura de datos a través de diferentes aplicaciones y también sirve para estructurar, almacenar e intercambiar información (W3C España, 2015). XML define la sintaxis y los requisitos que deben cumplir los lenguajes de marcado, así mismo, especifica, organiza y permite nuevas formas de incluir información extra, a través de marcas, a nuestra información. En consecuencia, XML ofrece independencia de los datos respecto de las aplicaciones (Gutiérrez, 2001).

El presente documento se divide en 6 secciones: la sección 2, describe los antecedentes, origen e historia de WaterML; en la sección 3, se detallan las especificaciones básicas del estándar y su relación con otros estándares; en la sección 4, se estudia específicamente el documento WaterML 2.0 Part 1-Timeseries y se indica su relación con O\&M, GML, UML y XML; en la sección 5, se exponen los resultados y discusiones, y se finaliza el documento con la sección 6 de conclusiones.

\section{Antecedentes, Origen e Historia de WaterML}

WaterML 2.0 se realiza previo a un informe (Taylor, 2010) de investigación de estándares existentes en varios países como iniciativa para el intercambio de información hidrológica. Uno de los estándares investigados fue WaterML 1.0 (1.1) que fue desarrollado por Consortium of Universities for Advancement of Hydrologic Science Incorporated (CUAHSI) para su uso en los EE.UU.

\subsection{WaterML1.0 (1.1)}

WaterML fue desarrollado por CUAHSI (Zaslavsky, Valentine y Whiteaker, 2007) como un formato de intercambio de series temporales de datos hidrológicos. El nombre inicial se continúa usando para la versión posterior del OGC. Se han desarrollado diversas aplicaciones e implementaciones, como el software HydroDesktop y el sistema CUAHSI HIS (Ames et al, 2012; Whiteaker y Tarboton, 2012; McEnery, 2013) o el paquete WaterML R que combina los software R e HydroServer (Kadlec, 2015). 


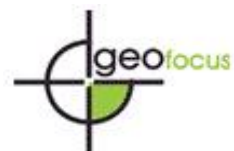

Salas Challco B. M., Masó Pau, J. y Pesquer Mayos, L. (2017): "Estudio del estándar WaterML”, GeoFocus (Artículos), $n^{o}$ 19, p. 129-150. ISSN: 1578-5157 http://dx.doi.org/10.21138/GF.485

\subsection{WaterML 2.0}

WaterML 2.0 del OGC, es un modelo de información armonizado entre múltiples estándares existentes del OGC (Khattar y Ames, 2014). Es un estándar abierto del OGC, es decir está disponible libremente para que cualquiera lo utilice con la finalidad de mejorar el intercambio de datos en el mundo. WaterML 2.0 es una versión actualizada de WaterML, se basa en el estándar O\&M de OGC (Almoradie et al, 2013).

La principal diferencia entre WaterML y WaterML 2.0 es en la forma de estructuración y la sintaxis. Además, WaterML 1.0 (1.1) utiliza como servicio web WaterOneFlow (McEnery et al, 2013; Zaslavsky et al., 2007) y WaterML 2.0 puede utilizar como servicio web el estándar SOS de OGC, así como otros servicios que proveen datos como Web Feature Service (WFS).

WaterML 2.0 se estructura en partes; la primera parte del estándar WaterML2.0: Timeseries se centra en un modelo de información estándar y una codificación XML derivada de este modelo para series temporales de observaciones hidrológicas. La segunda parte de WaterML 2.0: Ratings, Gaugings and Sections describe un modelo de información para intercambiar algunos indicadores y medidas observacionales muy específicas como por ejemplo secciones de ríos (Serra, 2015).

Otras partes del estándar WaterML 2.0 se extenderán a otras áreas de datos hidrológicos, tales como la descripción de calificación de curvas, información de calibración, diccionarios (controled vocabularies) etc.

Algunos estudios e implementaciones utilizan el estándar WFS como servidor de datos WaterML 2.0 (Kattar y Ames, 2014), también la combinación de los estándares WaterML 2.0 y SOS (Hussain, 2015), otras por ejemplo realizan modificaciones en el código o alternativas utilizando el método WaterML2.0-GeoServer con el servidor GeoServer (Almoradie et al, 2013), también existen los estudios de adaptación de Water Data Transfer Format (WDTF) hacia WaterML 2.0 (Car y Moore, 2011; Walker et al, 2009), así mismo las que utilizan tecnologías ESRI (Sijbertsma y Hodge, 2013) o por ejemplo GEOSS interoperability for weather, ocean and water (GEOWOW) que participó en el grupo WaterML 2.0 SWG (Vaccari, Craglia y Borzacchiello, 2013) para el avance de WaterML2.0-Part2 (Sheahan y Taylor, 2014), adicionalmente otros estudios siguen los pasos de WaterML y se crea otros estándares como es el caso de RiverML: A Harmonized Transfer Language for River Hydraulic Models (Jackson, 2014; Geoconnexion, 2013).

\subsubsection{WaterML 2.0 Part 1 - Timeseries}

WaterML 2.0 Part 1 (Taylor, 2014) describe series de tiempo como resultado de observaciones directas y datos procesados de una base de datos. WaterML2.0 se basa en la norma O\&M de la OGC (ISO 19156), y por lo tanto es significativamente diferente de CUAHSI WaterML.

\subsubsection{WaterML 2.0 Part 2 - Ratings, Gaugings and Sections}

WaterML 2.0 Parte 2 (Taylor, 2015) describe un modelo de información para intercambiar tablas de medidas, observaciones de indicadores y secciones de ríos. 


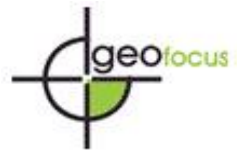

Salas Challco B. M., Masó Pau, J. y Pesquer Mayos, L. (2017): "Estudio del estándar WaterML”, GeoFocus (Artículos), $n^{\circ}$ 19, p. 129-150. ISSN: 1578-5157 http://dx.doi.org/10.21138/GF.485

\section{Especificaciones de WaterML y Estándares Relacionados}

\subsection{Conceptos básicos}

Los estándares web son un conjunto de recomendaciones dadas por la World Wide Web Consortium (W3C), OGC, entre otras organizaciones internacionales acerca de cómo crear e interpretar documentos basados en la web. El objetivo es crear una web accesible al mayor número de usuarios y que funcione en cualquier dispositivo con acceso a internet (Figura 1).

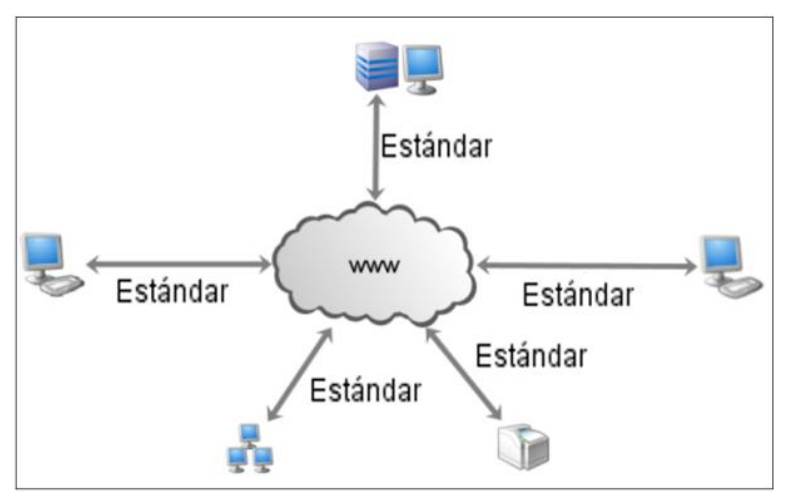

Figura1. Distintos dispositivos y redes comunicándose a través de protocolos y lenguajes comunes.

Fuente: http://www.w3c.es/Divulgacion/GuiasBreves/Estandares

Los estándares y documentos de apoyo de OGC son documentos técnicos que detallan interfaces informáticas o formas de codificación de datos. Los desarrolladores de software utilizan estos documentos para construir interfaces abiertas y codificaciones en sus productos y servicios (Open Geospatial Consortium, 2015). Es preciso recordar que WaterML 2.0 está implementado como un esquema de aplicación de acuerdo a las reglas del estándar GML (Figura 2).

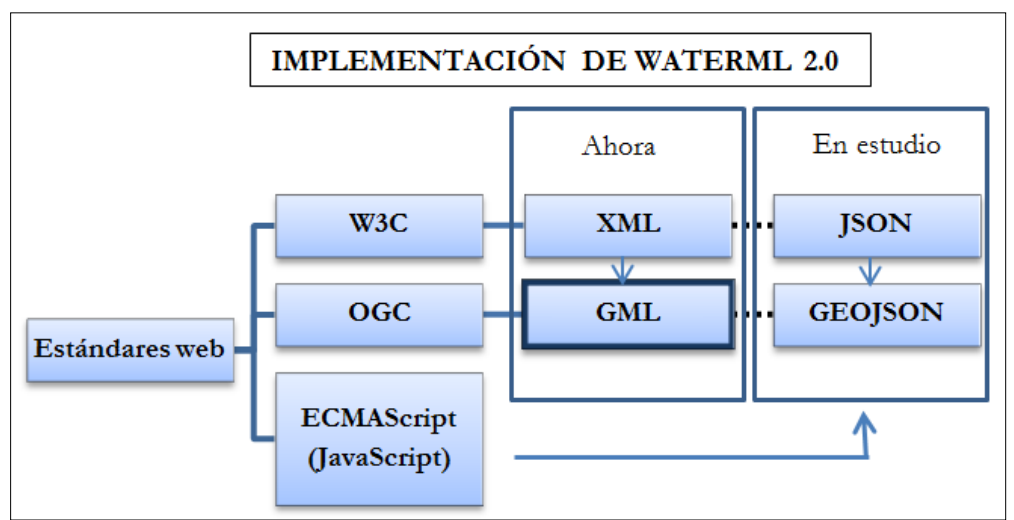

Figura 2: Diagrama de relación entre los principales estándares web e implementación de WaterML 2.0 de acuerdo a las reglas GML.

Fuente: Elaboración propia.

Los estándares OGC se pueden categorizar en estándares de codificación y datos, de servicios web, de programación de aplicaciones (Application Program Interface-API), y para clientes web (Bermúdez, Masó y Capdevila, 2012). 


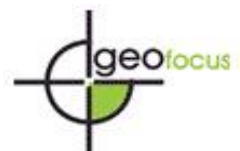

Salas Challco B. M., Masó Pau, J. y Pesquer Mayos, L. (2017): "Estudio del estándar WaterML", GeoFocus (Artículos), $n^{\circ}$ 19, p. 129-150. ISSN: 1578-5157 http://dx.doi.org/10.21138/GF.485

Un formato se entiende como la forma y modalidad de almacenamiento de un archivo en una computadora. Por ejemplo, el formato .mp3 (de música o audio), .doc (de texto), .xml (de web), .gml (de sistemas geográficos).

Un servicio web es un conjunto de aplicaciones o tecnologías con capacidad de operar en la web. Los servicios web interoperables proporcionan mecanismos de comunicación con estándares entre diferentes aplicaciones, interactuando entre sí para presentar información dinámica al usuario. Por ejemplo: WFS (servicio web para datos vectoriales geográficos), SOS (interfaz para sistemas de sensores).

Un modelo conceptual de datos en términos de la programación orientada a objetos es la representación del mundo real en objetos que se clasifican en clases (Canchala, 2015). WaterML 2.0 utiliza como modelo el Unified Modeling Language (UML) que es una manera gráfica de expresar un modelo conceptual de datos y a XML como una forma de almacenar información semántica.

XML Schemas (esquemas XML), son una forma de definir vocabularios específicos que serán aplicados a un documento XML. El término esquemas se ha tomado del mundo de las bases de datos relacionales donde un esquema se refiere al modelo al que un conjunto de datos se ajustan (Gutierrez et al, 2001). XML provee una manera de definir esquemas. Por ejemplo:

- El estándar O\&M especifica una implementación XML para las observaciones y mediciones.

- El estándar WaterML 2.0 está basado en O\&M que también es un esquema de aplicación de acuerdo a GML.

Finalmente, se entiende como encoding (codificación) al proceso de convertir los datos en un formato requerido para una serie de necesidades de procesamiento de información.

\subsection{Estándares relacionados}

\subsubsection{WFS}

El estándar Web Feature Service (WFS) es un servicio web y permite el acceso a datos vectoriales GML, representa un cambio en la forma en que se crea la información geográfica, se modifica e intercambia en Internet. En lugar de compartir la información geográfica a nivel de archivos mediante el protocolo de transferencia de archivos (FTP), por ejemplo, WFS ofrece acceso directo a la información geográfica como los ficheros (Vretanos, 2010).

3.2.2. GML

El estándar Geographic Markup Language (GML) es un estándar internacional extensible para el intercambio de datos espaciales (Portele, 2012). GML es un formato de datos geográficos.

\subsubsection{SOS}

El estándar Sensor Observation Service (SOS) proporciona una interfaz estandarizada para la gestión y acceso de metadatos y observaciones de sensores (Bröring, Stasch y Echterhoff, 2012). SOS es un servicio web para archivos O\&M.

\subsubsection{O\&M}

El estándar Observations and Measurements (O\&M) es utilizado para la descripción de las observaciones y las relaciones con los diversos aspectos importantes del proceso de 


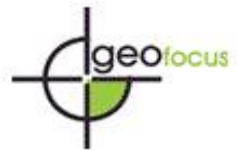

Salas Challco B. M., Masó Pau, J. y Pesquer Mayos, L. (2017): "Estudio del estándar WaterML", GeoFocus (Artículos), $n^{\circ}$ 19, p. 129-150. ISSN: 1578-5157 http://dx.doi.org/10.21138/GF.485

observación y para definir un esquema XML que puede utilizarse para el intercambio de observaciones (Cox, 2011; Cox, 2013). O\&M es un modelo conceptual y también define una codificación XML que sirve para resultados de observaciones SOS y para observaciones específicas como WaterML 2.0 para observaciones hidrológicas.

\subsubsection{SensorML}

El estándar Sensor Model Language (SensorML) tiene como objetivo principal permitir la interoperabilidad en el nivel sintáctico y semántico de modo que los sensores y los procesos puedan ser mejor entendidos por las máquinas, utilizadas de forma automática en los flujos de trabajo complejos, y fácilmente compartidos entre nodos web de sensores inteligentes (Botts y Robin, 2014). SensorML es un modelo conceptual y un esquema XML para la descripción de los sensores. En la Tabla 1 se puede observar la relación entre los conceptos y los estándares.

Tabla 1: Relación entre conceptos y estándares.

\begin{tabular}{|l|l|}
\hline \multicolumn{1}{|c|}{ Conceptos } & \multicolumn{1}{c|}{ Estándares } \\
\hline Codificaciones / Esquemas XML & O\&M, SensorML, GML, WaterML 2.0 \\
\hline Servicios Web & SOS, WFS \\
\hline Modelo Conceptual & O\&M, SensorML, ISO 19109 \\
\hline Formato de Datos & XML, JSON \\
\hline Formato de Datos Geográficos & GML, GeoJSON \\
\hline
\end{tabular}

Fuente: Elaboración propia.

El estándar O\&M sirve para observaciones generales, WaterML 2.0 es una extensión de éste que específicamente sirve para observaciones hidrológicas. Para acceder a estos datos es posible utilizar el servicio web SOS que permite trabajar con datos O\&M e incluso desde sensores modelizados como SensorML La localización del sensor se describe en GML, y puede ser proporcionado por un servicio WFS que permite el acceso a datos vectoriales en formato GML. En la Figura 3 se observan todas estas interconexiones.

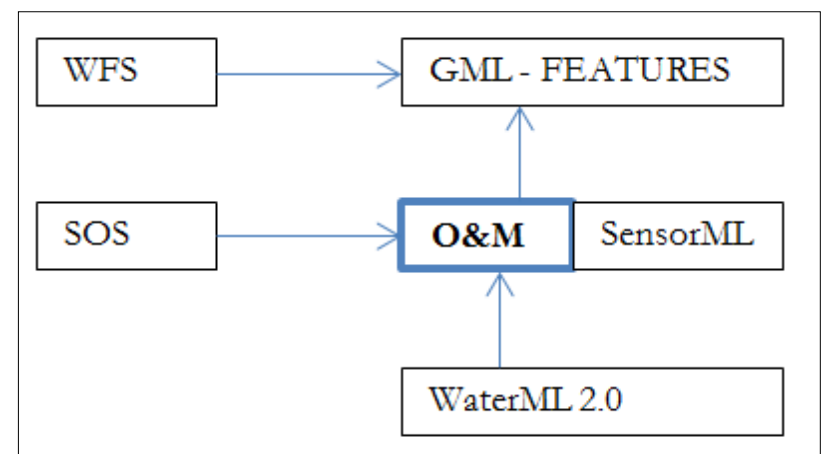

Figura 3: Diagrama de relación entre los principales estándares relacionados al estándar WaterML 2.0

Fuente: Elaboración propia. 
Salas Challco B. M., Masó Pau, J. y Pesquer Mayos, L. (2017): "Estudio del estándar WaterML", GeoFocus (Artículos), $n^{\circ}$ 19, p. 129-150. ISSN: 1578-5157 http://dx.doi.org/10.21138/GF.485

\section{Documento de estudio}

El presente estudio se centra básicamente en la parte 1 del estándar WaterML 2.0 Part 1-Timeseries, con el foco en la descripción de series de tiempo como resultado de observaciones directas y los datos procesados, así como las previsiones y resultados derivados.

\subsection{Métodos}

WaterML 2.0 - Part 1 - Timeseries ha sido implementado en dos grandes secciones (veáse Figura 4):

- La primera consiste en un modelo conceptual UML para datos de observación especificado con el perfil Observations \& Measurements O\&M.

- La segunda fue desarrollada para la implementación del modelo conceptual UML en XML, específicamente en el esquema XML de GML 3.2.

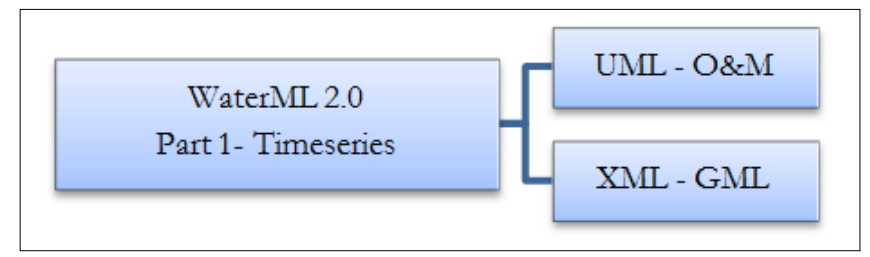

Figura 4. Diagrama de división de la documentación del estándar de la OGC WaterML 2.0-Part 1- Timeseries.

Fuente: Elaboración Propia.

\subsubsection{UML - O\&M}

En la figura 5 se muestra los elementos necesarios para definir una observación genérica según el modelo conceptual UML de O\&M, y en la figura 6 se observa el mismo diagrama utilizando como ejemplo el proceso para una observación hidrológica. La tabla 2 representa la relación entre los componentes y conceptos. 
Salas Challco B. M., Masó Pau, J. y Pesquer Mayos, L. (2017): "Estudio del estándar WaterML”, GeoFocus (Artículos), $n^{\circ}$ 19, p. 129-150. ISSN: 1578-5157 http://dx.doi.org/10.21138/GF.485

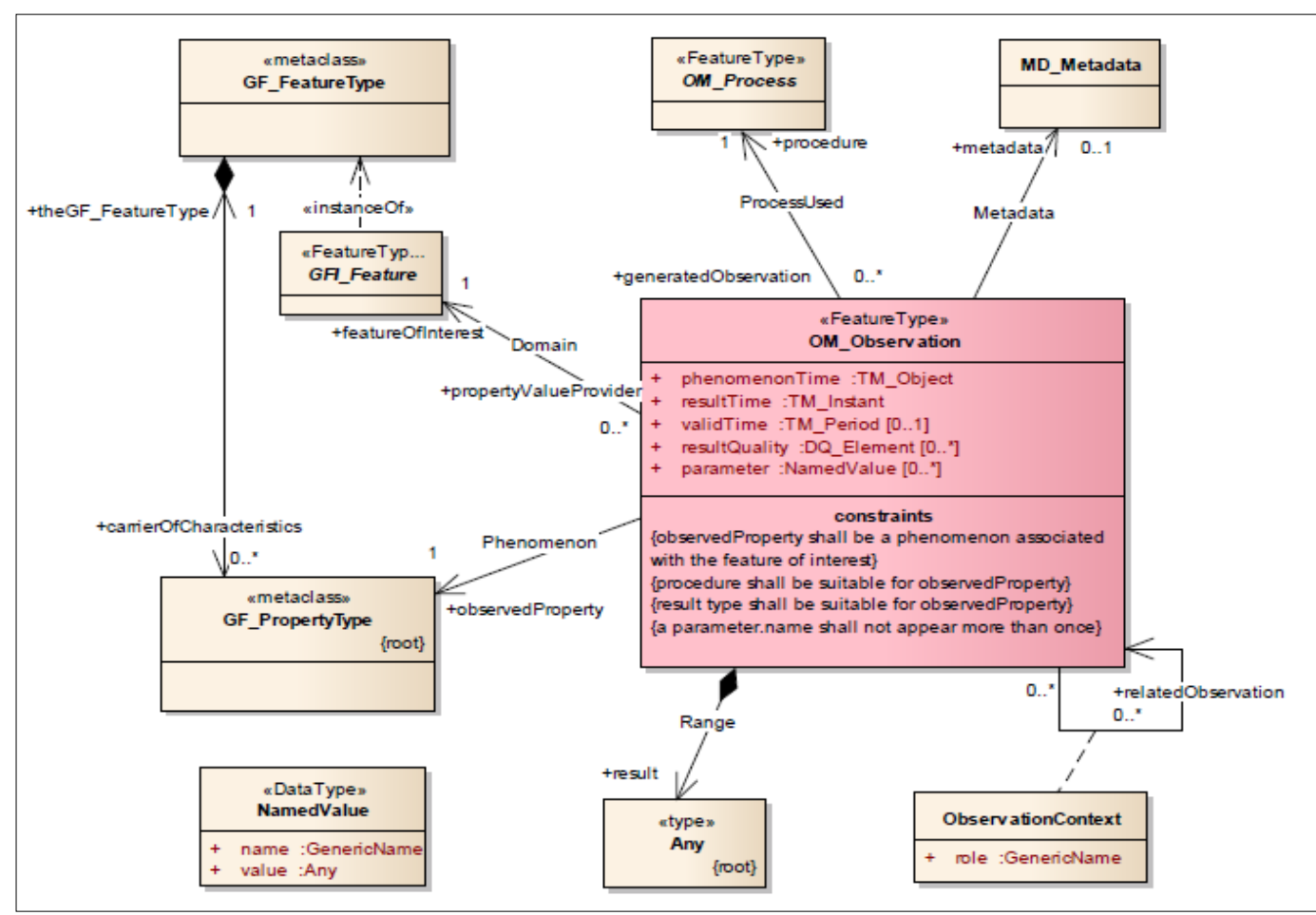

Figura 5: Diagrama en UML de una observación definida por O\&M Fuente: OGC-WaterML 2.0 -Part1 Timeseries

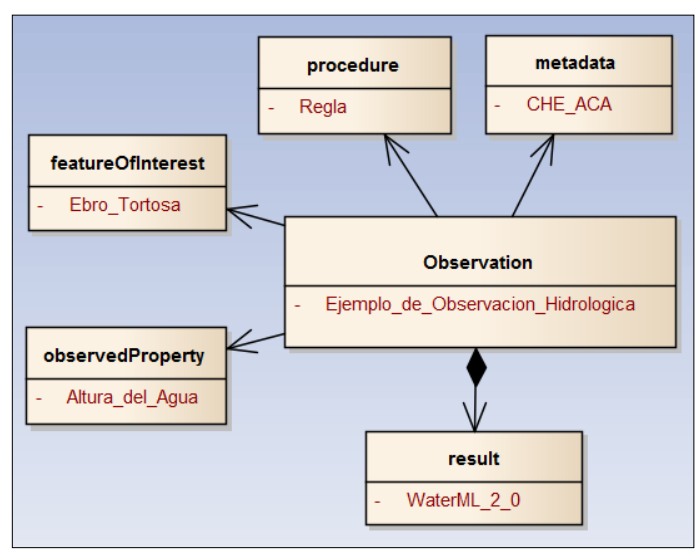

Figura 6: Ejemplo de una observación hidrológica utilizando el modelo conceptual de O\&M.

Fuente: Elaboración propia.

En el diagrama de la Figura 6 se observa la clase (Observation) con un ejemplo de observación hidrológica, en la clase (featureofInterest) la posición del sensor en el río Ebro de Tortosa, en la clase (observedProperty) la propiedad observada es la altura del agua, para medirla se utiliza una regla (procedure); la fuente de datos es CHE-ACA (metadata) y para que finalmente procese el resultado le pedimos que nos entregue en el estándar WaterML 2.0 (result). 


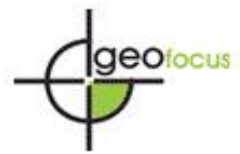

Revista Internacional de Ciencia y Tecnología de la In formación Geográfica International Review of Geographical Information Science and Technology

Salas Challco B. M., Masó Pau, J. y Pesquer Mayos, L. (2017): "Estudio del estándar WaterML”, GeoFocus (Artículos), $n^{\circ}$ 19, p. 129-150. ISSN: 1578-5157 http://dx.doi.org/10.21138/GF.485

Tabla 2. Relación de componentes de WaterML 2.0 y O\&M 2.0

\begin{tabular}{|l|l|}
\hline \multicolumn{1}{|c|}{ WaterML 2.0 components } & \multicolumn{1}{c|}{ O\&M 2.0 concepts } \\
\hline Time series & Result \\
\hline Observation specialisations & Observation \\
\hline $\begin{array}{l}\text { Procedures used in } \\
\text { measurement/analysis/processing }\end{array}$ & Procedure \\
\hline Observation metadata & Observation (metadata) \\
\hline Location description & Sampling features \\
\hline Collections & - \\
\hline & \\
\hline
\end{tabular}

Fuente: OGC-WaterML 2.0 Part1 Timeseries.

En esta parte del estudio, se definen las siguientes clases de WaterML 2.0:

- Timeseries Observation (abstract): Es una clase abstracta que captura las restricciones comunes de la clase OM_Observation de O\&M, para observaciones que tienen Timeseries como resultado.

- Timeseries Observation (domain range): Es una observación que retorna Timeseries con el tiempo y valor representados.

- Timeseries Observation (interleaved): Es una observación que retorna un Timeseries - timevalue pair coupled. Las dos clases más específicas son MeasurementTimeseriesTVPObservation y CategoricalTimeseriesTVPObservation que capturan los Timeseries especializados donde el tipo de valor está restringido.

- Timeseries (core): Una clase abstracta que captura las características comunes de los Timeseries.

- Timeseries (interleaved): Describe la estructura de interleaved Timeseries (time-value-pairs) y las clases de los metadatos asociados. Las dos clases más específicas (MeasurementTimeseriesTVP y CategoricalTimeseriesTVP) que sirven para capturar dos tipos específicos de Timeseries en el que el tipo de valor es restringido.

- Monitoring Points: Se utiliza para describir los puntos de muestreo utilizando O\&M.

- Monitoring point feature of interest: Clase para observaciones Timeseries utilizando puntos de monitoreo (in situ). Es una clase separada con el fin de permitir que las observaciones Timeseries utilicen diferentes características de muestreo (por ejemplo, pozos de agua subterránea) o con características más complejas geométricamente tales como líneas y polígonos.

- Collections: Se utiliza para proporcionar colecciones de sampling features, Timeseries, inline vocabularies.

- Observation Process: Se utiliza para los procesos descritos involucrados en la generación de Timeseries.

\subsubsection{XML - GML}

Los esquemas XML fueron autogenerados a partir de las reglas de codificación de GML 3.2 (Portele, 2007), que a su vez también fueron definidos por en un modelo conceptual UML. Estos esquemas fueron modificados para cumplir con los requisitos señalados. Los patrones Schematron (ISO/IEC 19757-3, 2006) también se implementaron para requisitos adicionales donde correspondían. El lenguaje Schematron forma parte del estándar ISO-DSDL (Document Schema Definition Languages). La función de este lenguaje es la validación de estructura y contenidos en documentos XML (Hedler, Montero y Kutscherauer, 2011). Estas reglas Schematron se utilizan en el servicio de validación WaterML 2.0 ( $\mathrm{Yu}$ et al, 2015, Yu et al 2012). 


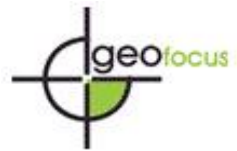

Salas Challco B. M., Masó Pau, J. y Pesquer Mayos, L. (2017): "Estudio del estándar WaterML", GeoFocus (Artículos), $n^{\circ}$ 19, p. 129-150. ISSN: 1578-5157 http://dx.doi.org/10.21138/GF.485

\section{A. Ejemplo del código XML}

Para comprender el modelo conceptual UML y XML de WaterML 2.0, se ha utilizado un ejemplo básico en XML con codificación (wml2) obtenido de $52^{\circ}$ North SOS Test Client, se describe el procedimiento de la solicitud al servicio web (del Paso 1 al 3) y la entrega de la respuesta codificada en WaterML 2.0 (en el Paso 4).

Paso 1: Ingresamos a la aplicación web de $52^{\circ}$ North SOS Test Client en la siguiente

URL: http://sensorweb.demo.52north.org/52n-sos-webapp/client

Paso 2: Solicitamos a la aplicación web que nos entregue el resultado en WaterML 2.0 indicando en la solicitud (GetObservation) y la respuesta (responseFormat) (http://www.opengis.net/WaterML/2.0).

Codificación para que la respuesta esté en WaterML 2.0: <sos: responseFormat>http://www. opengis.net/WaterML/2.0</sos: responseFormat>

Paso 3: Finalmente ingresamos la solicitud al servicio web del SOS.

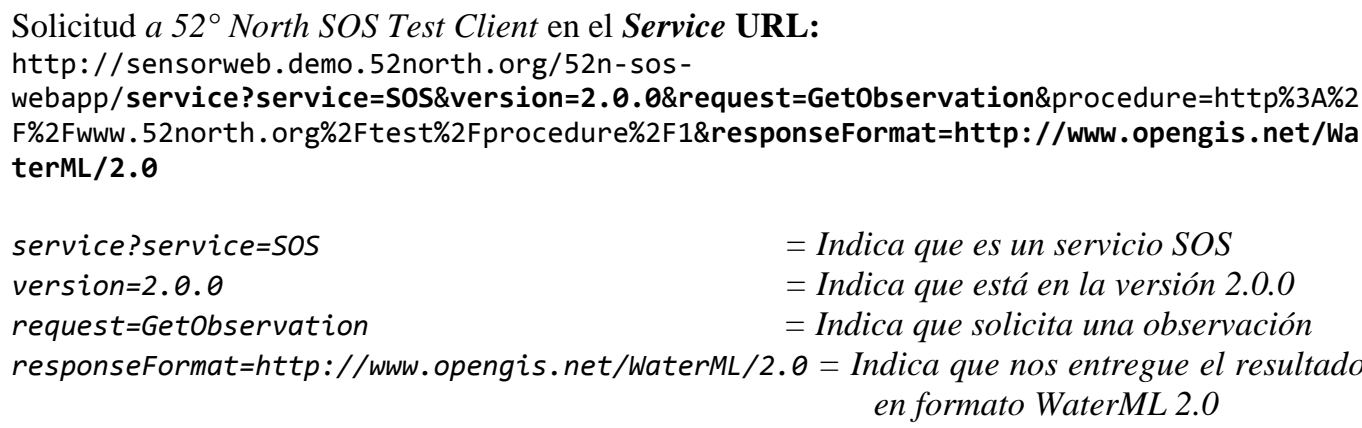

Paso 4: El servicio web SOS $52^{\circ}$ North SOS Test Client, entrega el resultado en XML con codificación WaterML 2.0, se explica partes de las Líneas de Código Fuente (LCF) en la tabla 3.

1. <?xml version="1.0" encoding="UTF-8"?>

2. <sos:GetObservationResponse xmlns:sos="http://www.opengis.net/sos/2.0" xmlns:xsi="http://www.w3.org/2001/XMLSchema-instance"

xmlns:om="http://www.opengis.net/om/2.0" xmlns:gml="http://www.opengis.net/gml/3.2" xmlns:xlink="http://WwW.w3.org/1999/xlink" xmlns:wml2="http://www. opengis.net/waterml/2.0"

xsi:schemaLocation="http://www.opengis.net/waterml/2.0 http://schemas.opengis.net/waterml/2.0/waterml2.xsd http://www.opengis.net/sos/2.0 http://schemas.opengis.net/sos/2.0/sosGetObservation.xsd http://www.opengis.net/waterml-dr/2.0 http://schemas.opengis.net/waterml/2.0/domainrange-informative/timeseries-domain-range.xsd http://www.opengis.net/gml/3.2 http://schemas.opengis.net/gml/3.2.1/gml.xsd http://www.opengis.net/gmlcov/1.0 http://schemas.opengis.net/gmlcov/1.0/gmlcovAll.xsd http://www.opengis.net/om/2.0 http://schemas.opengis.net/om/2.0/observation.xsd">

3. 〈sos:observationData>

4. 〈om:OM_Observation gml:id="o_63425FF441F4AB8307AAE0673886AA882CD81298">

5. <om:phenomenonTime>

6. 〈gml:TimePeriod gml:id="phenomenonTime_1">

7. <gml:beginPosition>2012-11-19T13:00:00.000Z</gml:beginPosition>

8. <gml:endPosition>2012-11-19T13:09:00.000Z</gml:endPosition>

9. </gml:TimePeriod>

10. 〈/om:phenomenonTime>

11. 〈om:resultTime> 
Salas Challco B. M., Masó Pau, J. y Pesquer Mayos, L. (2017): "Estudio del estándar WaterML”, GeoFocus (Artículos), $n^{\circ}$ 19, p. 129-150. ISSN: 1578-5157 http://dx.doi.org/10.21138/GF.485

12. 〈gml:TimeInstant gml:id="ti_3AD9AE058FCA54D35609386C41EDDCE2A1F505C3">

13. 〈gml:timePosition>2012-11-19T13:01:00.000Z</gml:timePosition>

14. 〈/gml:TimeInstant>

15. </om:resultTime>

16. <om:procedure xlink:href="http://www.52north.org/test/procedure/1"/>

17. <om:observedProperty xlink:href="http://www.52north.org/test/observableProperty/1"/>

18. <om:featureOfInterest xlink:href=|"http://www.52north.org/test/featureOfInterest/1"/>

19. 〈om:result>

20. 〈wml2:MeasurementTimeseries gml:id="timeseries.1"〉

21. 〈wm12:metadata>

22. 〈wml2:TimeseriesMetadata>

23. <wml2: temporalExtent xlink:href="\#phenomenonTime_1"/>

(Línea 18) Posición/lugar de interés.

24. </wml2:TimeseriesMetadata>

25. </wml2: metadata $>$

26. 〈wml2: defaultPointMetadata>

27. 〈wm12:DefaultTVPMeasurementMetadata>

28. <wml2: uom code="test_unit_1"/>

29. <wml2: interpolationType

xlink:href="http://wWW.opengis.net/def/timeseriesType/WaterML/2.0/continuous" xlink:title="Continuous" / >

30. </wml2: DefaultTVPMeasurementMetadata $>$

31. </wml2: defaultPointMetadata $>$

32. 〈wml2: point>

33. 〈wml2: MeasurementTVP>

34. 〈wml2: time>2012-11-19T13:00:00.000Z</wm12:time>

35. 〈wml2: value $>1.0</$ wml2:value $>$

36. $\langle/$ wml2: MeasurementTVP>

37. </wml2: point >

38. 〈wml2:point>

39. 〈wm12:MeasurementTVP>

40. 〈wml2: time>2012-11-19T13:01:00.000Z</wml2:time>

41. 〈wml2: value $>1.1</$ wml2: value $>$

42. 〈/Wml2: MeasurementTVP>

43. </wm12:point $>$

Tabla 3. Explicación de código fuente WaterML 2.0

\begin{tabular}{|c|c|c|}
\hline LCF & Código Fuente & Explicación \\
\hline 2 & <sos:GetObservationResponse & $\begin{array}{l}\text { Indica que es una respuesta a una petición SOS de tipo } \\
\text { GetObservation. }\end{array}$ \\
\hline 3 & $\langle$ sos:observationData〉 & Indica que nos muestra unos datos (ObservationData). \\
\hline \multirow{2}{*}{4} & <om: & Es el espacio de nombres (namespace) de O\&M. \\
\hline & OM_Observation & Nos muestra que tenemos las observaciones. \\
\hline 5 & 〈om:phenomenonTime> & $\begin{array}{l}\text { Nos indica que las observaciones son tomadas en un } \\
\text { tiempo. }\end{array}$ \\
\hline \multirow[b]{2}{*}{6} & <gml:TimePeriod & Especifica el periodo de tiempo. \\
\hline & gml:id="phenomenonTime_1> & $\begin{array}{l}\text { Indica a la referencia "xlink:href="\#phenomenonTime_1" de la } \\
\text { línea } 23 .\end{array}$ \\
\hline 7 & $\begin{array}{l}\text { <gml:beginPosition>2012-11- } \\
\text { 19T13:00:00.000Z }\end{array}$ & \multirow{2}{*}{$\begin{array}{l}\text { Indican los valores de inicio y fin del periodo, por ejemplo } \\
\text { en este caso sería } 9 \text { minutos de diferencia para el mismo } \\
\text { día. }\end{array}$} \\
\hline 8 & $\begin{array}{l}\langle\mathrm{gml} \text { : endPosition>2012-11- } \\
\text { 19T13:09:00.000Z }\end{array}$ & \\
\hline 11 & 〈om:resultTime> & Indica la respuesta en un tiempo. \\
\hline 13 & $\begin{array}{l}\langle\mathrm{gml}: \text { timePosition }>2012-11- \\
\text { 19T13:01:00.000Z</gml:timePositi } \\
\text { on> }\end{array}$ & $\begin{array}{l}\text { Indica la respuesta en una posición de tiempo determinado } \\
\text { en 13:01:00. }\end{array}$ \\
\hline 19 & $\langle$ om:result $>$ & Indica el resultado. \\
\hline 20 & $\begin{array}{l}\langle\text { wm12:MeasurementTimeseries } \\
\text { gml:id="Timeseries .1"> }\end{array}$ & $\begin{array}{l}\text { Indica que está codificada en WaterML } 2.0 \text { y es una } \\
\text { Timeseries. }\end{array}$ \\
\hline 21 & $\langle$ wml2:metadata $\rangle$ & Indica que tiene metadatos. \\
\hline
\end{tabular}


Salas Challco B. M., Masó Pau, J. y Pesquer Mayos, L. (2017): "Estudio del estándar WaterML”, GeoFocus (Artículos), $n^{o}$ 19, p. 129-150. ISSN: 1578-5157 http://dx.doi.org/10.21138/GF.485

\begin{tabular}{|c|c|c|}
\hline 23 & $\begin{array}{l}\text { <wml2: temporalExtent } \\
\text { xlink: href="\#phenomenonTime_1" / }\end{array}$ & Indica que es una extensión temporal. \\
\hline 28 & $\langle$ 〈wm12:uom code="test_unit_1"/> & Indica el código de las unidades de medida. \\
\hline 29 & $<$ wm12: interpolationType & $\begin{array}{l}\text { Indica que hay un sistema de interpolación continua y la } \\
\text { extraemos del vocabulario definido en WaterML2.0. }\end{array}$ \\
\hline 32 & $\langle w m 12$ :point $\rangle$ & Indica los puntos de la Timeseries. \\
\hline 33 & $\langle$ wm12:MeasurementTVP $\rangle$ & $\begin{array}{l}\text { Indica que cada punto está formado por una medida (TVP; } \\
\text { Time-Value-Pairs). }\end{array}$ \\
\hline 34 & $\begin{array}{l}\langle\text { wm12: time }>2012-11- \\
\text { 19T13:00:00.000Z</wm12:time> }\end{array}$ & Indica el tiempo del primer par o punto 1 de la Timeseries. \\
\hline 35 & $\langle$ wm12:value $\rangle 1.0</$ wml2:value $\rangle$ & Indica el valor del primer par o punto que es 1.0. \\
\hline
\end{tabular}
Fuente: Elaboración propia.

\section{Resultados y discusión}

Para la demostración del presente estándar se ha realizado una extensa búsqueda en bibliografía (último acceso en 30/07/2015) y recursos web sobre herramientas, aplicaciones web o de escritorio, las cuales hacen referencia o hacen uso del estándar WaterML en su versión 1.0 (1.1), 2.0 Part 1 Timeseries, 2.0 Part 2 Ratings, Gaugings and Sections. En la Tabla 4, se detalla un amplio abanico de ejemplos. El URL de cada ejemplo se ha probado con los principales navegadores como son Google Chrome, Internet Explorer y Opera, respondiendo en todas ellas.

Tabla 4: Portales web y algunos ejemplos encontrados asociados a WaterML APLICACIONES WEB

\begin{tabular}{|c|c|}
\hline \multicolumn{2}{|r|}{ APLICACIONES WEB } \\
\hline & WaterML $1.0(1.1)$ \\
\hline $\mathbf{N}^{\circ}$ & Institución: URL del Producto \\
\hline \multirow[t]{3}{*}{1} & USGS: http://waterservices.usgs.gov/nwis/iv/?sites=08158000\&period=P7D\&parameterCd=00060 \\
\hline & Nombre del Producto: USGS Water Service \\
\hline & Objetivo: Muestra ejemplos de código WaterML $1.0(1.1)$ \\
\hline \multirow{7}{*}{2} & WaterML 1.0 (1.1) y WaterML 2.0 Part 1 \\
\hline & \\
\hline & Nombre del Producto: GEOSS Water Services: Global Precipitation Map \\
\hline & (Salas, 2013) \\
\hline & Objetivo: Mapa de Precipitaciones Global, que se desarrolló como parte de la iniciativa \\
\hline & GEOSS Architecture Implementation Pilot (AIP-6). Al hacer clic en un punto, se tiene la \\
\hline & disponibilidad. Utiliza ESRI ArcGIS Online y la fuente de datos de GEOSS. \\
\hline \multirow{8}{*}{3} & WaterML 2.0 Part 1 \\
\hline & FLEMISH WATER MANAGERS: http://www.waterinfo.be/ \\
\hline & Nombre del Producto: WATERINFO.be - Portal of the Flemish Water managers \\
\hline & Objetivo: Informar con la mayor precisión posible sobre las inundaciones y las sequías a \\
\hline & través del portal web. Los informes contienen información actual publicados por $V M M$ and \\
\hline & Hydrological Information Centre (HIC) - Flanders Bélgica. La web permite visualizar las \\
\hline & Timeseries, permite la descarga de datos en formatos CSV, HTML, JSON y WaterML2.0. El \\
\hline & portal está en holandés, inglés y francés. Es un portal muy dinámico. \\
\hline \multirow{4}{*}{4} & 52 $^{\circ}$ NORTH: http://sensorweb.demo.52north.org/sensorwebclient-webapp-stable/ \\
\hline & Nombre del Producto: Sensor Web Open Source Client \\
\hline & $\begin{array}{l}\text { Objetivo: Permite seleccionar la fuente de datos, visualizar la posición de los sensores en el } \\
\text { mapa, buscar, ver las características de las Timeseries, para luego ser graficadas y exportadas }\end{array}$ \\
\hline & \\
\hline
\end{tabular}


Salas Challco B. M., Masó Pau, J. y Pesquer Mayos, L. (2017): "Estudio del estándar WaterML”, GeoFocus (Artículos), $n^{o}$ 19, p. 129-150. ISSN: 1578-5157 http://dx.doi.org/10.21138/GF.485

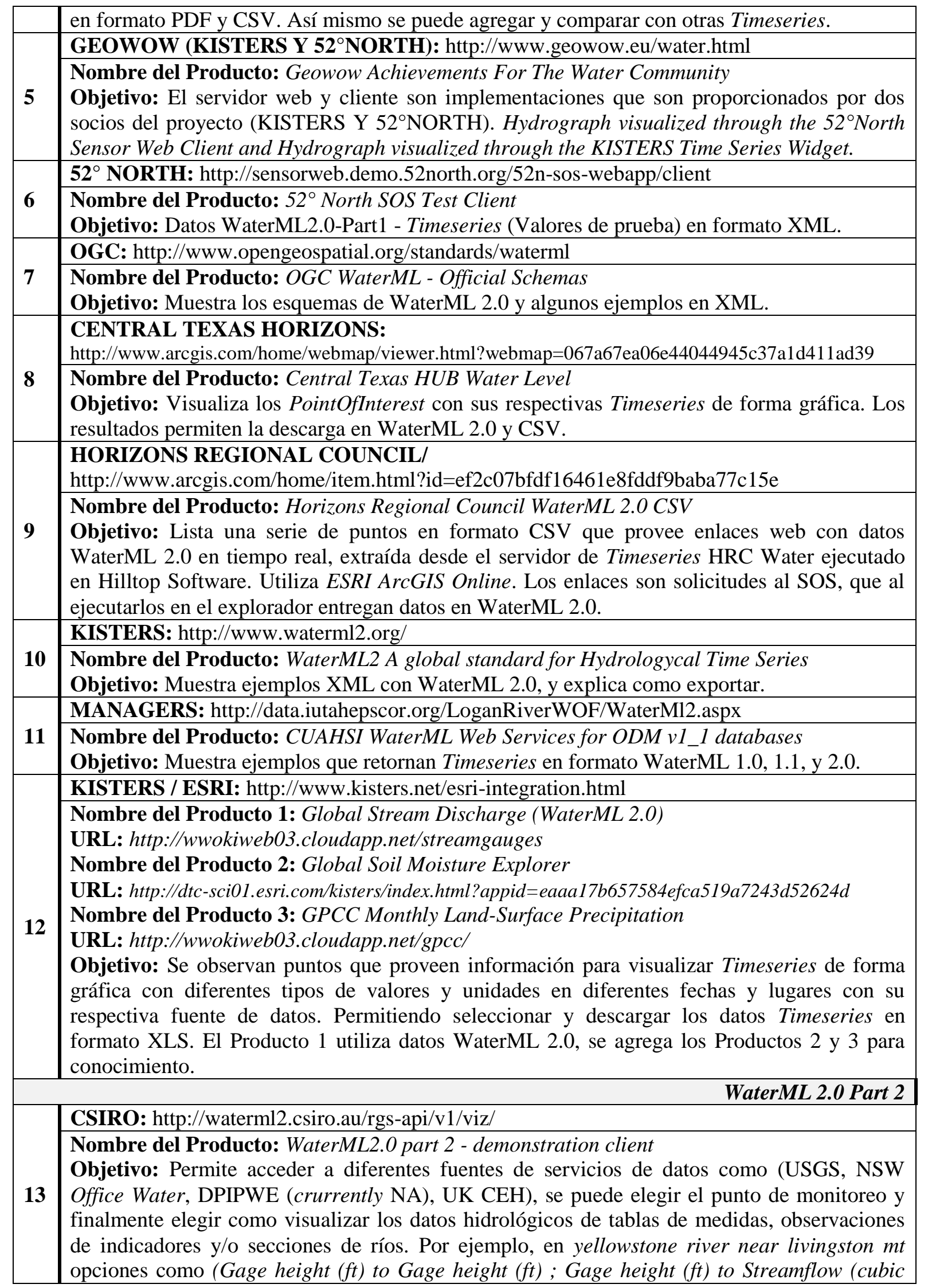


Salas Challco B. M., Masó Pau, J. y Pesquer Mayos, L. (2017): "Estudio del estándar WaterML”, GeoFocus (Artículos), $n^{\circ}$ 19, p. 129-150. ISSN: 1578-5157 http://dx.doi.org/10.21138/GF.485

\begin{tabular}{|c|c|}
\hline & $\mathrm{ft} / \mathrm{sec})$ \\
\hline & 7th Framework Programme of the European Union: http://www.waterp-fp7.eu/ \\
\hline 14 & $\begin{array}{l}\text { Nombre del Producto: Proyecto WatERP } \\
\text { Objetivo: WatERP desarrollará una web basada en Open Management Platform (OMP) } \\
\text { apoyado por el conocimiento en tiempo real sobre la oferta y la demanda del agua, lo que } \\
\text { permite a todo el sistema de distribución de agua ser visto de manera integral y personalizada. } \\
\text { Esta información será almacenada en WaterML 2.0. }\end{array}$ \\
\hline \multirow[b]{2}{*}{15} & CSIRO: http://waterml2.csiro.au/rgs-api/v1/gauging/18953/?format=xml \\
\hline & $\begin{array}{l}\text { Nombre del Producto: WaterML } 2.0 \text { part } 2 \text { RESTful API - Gauging List } \\
\text { Objetivo: Ejemplo y demostración del código WaterML } 2.0 \text {-Part } 2 \text { en XML para el punto de } \\
\text { monitoreo } 18953 \text {. El código también puede obtenerse en formato JSON. }\end{array}$ \\
\hline \multirow[b]{2}{*}{16} & CSIRO: http://waterml2.csiro.au/rgs-api/v1/?format=api \\
\hline & $\begin{array}{l}\text { Nombre del Producto: WaterML } 2.0 \text { part } 2 \text { RESTful API } \\
\text { Objetivo: This is a test API for the WaterML2.0 part } 2 \text { OGC Interoperability Experiment. No } \\
\text { gaurantees are made for its reliability or stability. The data contained within this API is test } \\
\text { data only, kindly supplied by the NSW Office of Water (http://www.water.nsw.gov.au/) } \\
\text { Muestra links con listado de ejemplos de datos hidrológicos disponibles en: } \\
\text { - Gauging list: "http://waterml2.csiro.au/rgs-api/vl/gauging/", } \\
\text { - ConversionList: "http://waterml2.csiro.au/rgs-api/vl/conversion/", } \\
\text { - Monitoring Points-List: "http://waterml2.csiro.au/rgs-api/vl/monitoring-point/" } \\
\text { Ingresando a estos links se pueden descargar los datos hidrológicos en formato API, JSON, } \\
\text { XML. }\end{array}$ \\
\hline \multirow[b]{2}{*}{17} & CSIRO: http://waterml2.csiro.au/validator/ \\
\hline & $\begin{array}{l}\text { Nombre del Producto: WaterML2.0 part } 2 \text { API validator } \\
\text { Objetivo: This service is provided for the operation of the WaterML2.0 part } 2 \text { Interoperability } \\
\text { Experiment. WaterML2.0 part } 2 \text { is a format for exchanging hydrological rating tables and } \\
\text { gaugings (observations). Se observa un ejemplo URL y punto de monitoreo ID. }\end{array}$ \\
\hline \multirow[b]{2}{*}{18} & NRFA /CEH: http://nrfa-ceh2.cloudapp.net:8080/52n-sos-dev/static/client/jsClient/\#map \\
\hline & $\begin{array}{l}\text { Nombre del Producto: Gauge daily flow del National River Flow Archive } \\
\text { Objetivo: Servicio SOS de observaciones hidrológicas en estaciones fluviales del Reino } \\
\text { Unido. Series temporales y localizaciones geográficas en formato WaterML 2.0. }\end{array}$ \\
\hline \multicolumn{2}{|r|}{ SOFTWARE/APLICACIONES DE ESCRITORIO } \\
\hline & WaterML 1.0 (1.1) \\
\hline \multirow[t]{2}{*}{19} & CUAHSI: $\underline{\text { http://his.cuahsi.org }}$ \\
\hline & $\begin{array}{l}\text { Nombre del Producto: CUAHSI's Hydrologic Information System (CUAHSI-HIS) } \\
\text { Objetivo: Sistema basado en internet para el intercambio de datos hidrológicos. Trabaja con } \\
\text { WaterML } 1.0 \text { (1.1). Los requisitos Hardware/Software están indicados en el manual del } \\
\text { software. }\end{array}$ \\
\hline \multirow[b]{2}{*}{20} & CUAHSI: http://his.cuahsi.org/hydroserver.html \\
\hline & $\begin{array}{l}\text { Nombre del Producto: HydroServer } \\
\text { Objetivo: Es parte del sistema HIS (Hydrologic Information System) que almacena y publica } \\
\text { datos hidrológicos. Es una de las } 3 \text { piezas principales del sistema HIS y se comunica con el } \\
\text { servicio web WaterOneFlow. } \\
\text { Algunos componentes principales: } \\
\text { - HydroServer Time Series Analyst Versión 1.1.3 } \\
\text { - HydroServer Website 1.1.3.2 } \\
\text { - HydroServer Map Web Application 1.1 } \\
\text { Trabaja con WaterML 1.0 (1.1). Debe instalarse previamente algunos productos comerciales } \\
\text { con licencia, p.ej: ESRI ArcGIS 9.3.1 Desktop, Microsoft SQL Server 2008, etc. }\end{array}$ \\
\hline 21 & CUAHSI: http://his.cuahsi.org/hydrodesktop.html \\
\hline
\end{tabular}




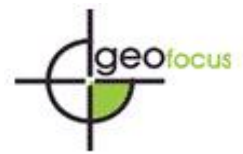

Salas Challco B. M., Masó Pau, J. y Pesquer Mayos, L. (2017): "Estudio del estándar WaterML”, GeoFocus (Artículos), $n^{\circ}$ 19, p. 129-150. ISSN: 1578-5157 http://dx.doi.org/10.21138/GF.485

\begin{tabular}{|c|c|}
\hline & $\begin{array}{l}\text { Nombre del Producto: HydroDesktop } \\
\text { Objetivo: Es un SIG de código libre, que contiene herramientas para utilizar datos } \\
\text { hidrológicos del sistema CUAHSI- HIS. Los requisitos de hardware / software están indicados } \\
\text { en el manual. Trabaja con datos WaterML } 1.0 \text { (1.1). }\end{array}$ \\
\hline \multirow[b]{2}{*}{22} & CUAHSI: http://his.cuahsi.org/documents/WaterMLv11Samples.zip \\
\hline & $\begin{array}{l}\text { Nombre del Producto: WaterML 1.1 Examples Files } \\
\text { Objetivo: Muestra ejemplos de WaterML 1.1. Se necesita un software para leer XML y } \\
\text { software de descompresión de datos. }\end{array}$ \\
\hline & CUAHSI: http://his.cuahsi.org/wofws.html \\
\hline 23 & $\begin{array}{l}\text { Nombre del Producto: WaterOneFlow Web Services \& WaterML } \\
\text { Objetivo: CUAHSI-HIS utiliza una familia de servicios web llamado WaterOneFlow que } \\
\text { retorna datos en formato WaterML 1.1. Así mismo la web brinda enlaces a ejemplos y } \\
\text { funcionalidades de WaterML }(1.0,1.1) \text {, fuente de datos para CUAHSI WaterOneFlow Data } \\
\text { Services, y otros servicios web WaterML adicionales. El manual está indicado en el enlace que } \\
\text { ofrece la web del producto. }\end{array}$ \\
\hline \multirow[b]{2}{*}{24} & CUAHSI: http://his.cuahsi.org/hydroexcel.html \\
\hline & $\begin{array}{l}\text { Nombre del Producto: HydroExcel: WaterOneFlow Excel Client } \\
\text { Objetivo: HydroExcel es una hoja de cálculo de Excel que proporciona acceso directo a los } \\
\text { servicios web WaterOneFlow. Los requisitos de Hardware/Software están indicados en el } \\
\text { manual. Trabaja con datos WaterML } 1.0(1.1) \text {. }\end{array}$ \\
\hline & WaterML 2.0 Part 1 \\
\hline \multirow[t]{2}{*}{25} & 52 ${ }^{\circ}$ NORTH: http://52north.org/330-user-friendly-and-efficient-sos-4-0 \\
\hline & $\begin{array}{l}\text { Nombre del Producto: } 52^{\circ} \text { North SOS } 4.0 .0 \text {. } \\
\text { Objetivo: SOS que permite administrar de cerca datos de los sensores en tiempo real. Indica } \\
\text { que soporta dominios opcionales como WaterML } 2.0 \text { y SOS } 2.0 \text { de la OGC. Se Indica los } \\
\text { requisitos en el manual del SOS } 4.0 .0 \\
\text { URL de descarga del SOS: http://52north.org/downloads/category/3-sos }\end{array}$ \\
\hline \multirow[t]{2}{*}{26} & Microsoft Office Professional Plus 2010: http://www.microsoft.com/office/2010/es/ \\
\hline & $\begin{array}{l}\text { Nombre del Producto: } \text { Microsoft } \circledast \text { Excel } \circledast 2010 \\
\text { Objetivo: Permite importar ficheros XML desde la cinta de opciones (Datos-desde web), } \\
\text { logrando que los datos con codificación WaterML 2.0-Partl-Timeseries se puedan colocar en } \\
\text { columnas y filas, y así poder utilizar otras herramientas de análisis como insertar gráficos a } \\
\text { partir de estas Timeseries. Este es un producto comercial. }\end{array}$ \\
\hline & Fuente: Elaboración Propia (Último acceso a las URL's: 30/07/2015). \\
\hline
\end{tabular}

Al realizar la prueba en los diferentes ejemplos encontrados se observa que la información hidrológica proporcionada (time-value pairs) muestra diferentes tipos de gráficos de Timeseries. Ahora bien, no se observa la implementación de las codificaciones del estándar WaterML 2.0, en todos los casos que mencionan su uso. En aquellos ejemplos de la Tabla 4 que sí consiguen observar y descargar la codificación en WaterML, éstos pueden ser utilizados y transportados en formatos adicionales como CSV, XLS, XML, JSON, siendo importante para la interoperabilidad con componentes de otras aplicaciones y para los desarrolladores de software. Al mismo tiempo, en algunos casos las aplicaciones web generan información útil en formato PDF a partir de estos datos, para su divulgación y entendimiento de otro tipo de usuario, diferente a los desarrolladores.

Para conocer a fondo como está estructurada WaterML2.0 Parte 1- Timeseries y saber todas sus bondades es necesario estudiar cada uno de los paquetes y clases definidos en el modelo conceptual UML (Páginas 15 al 71 del documento de estudio). 


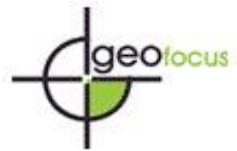

Salas Challco B. M., Masó Pau, J. y Pesquer Mayos, L. (2017): "Estudio del estándar WaterML", GeoFocus (Artículos), $n^{\circ}$ 19, p. 129-150. ISSN: 1578-5157 http://dx.doi.org/10.21138/GF.485

Es conveniente indicar que no se enunció sobre el uso de vocabularios controlados conocidos como code-lists, debido a que en el documento de estudio indican que WaterML 2.0 no define un conjunto completo de vocabularios para el intercambio de datos, lo hace solamente para las Timeseries, para lo cual prevén que las partes involucradas en el intercambio determinarán los vocabularios (Taylor, 2014; Yu et al, 2011). Así mismo mencionan que de manera óptima un organismo reconocido como la WMO gobernaría los vocabularios. Hydrology DWG tiene como trabajo futuro abordar el área de los vocabularios controlados para el dominio de la hidrología. Sin embargo existen estudios para aumentar la capacidad de vocabularios controlados para ser reutilizados por otros sistemas y aplicaciones (Yu et al, 2015).

La Tabla 5 constituye un resumen de la Tabla 4, y representa el cuadro global que indica la cantidad de recursos encontrados y en que versión de WaterML.

Tabla 5: Resumen de recursos para la demostración de WaterML

\begin{tabular}{|c|c|c|c|c|c|}
\hline \multirow{3}{*}{$\mathbf{N}^{\circ}$} & \multirow{3}{*}{ Recursos } & \multirow{3}{*}{ Uુ } & \multicolumn{3}{|c|}{ WaterML } \\
\hline & & & \multirow{2}{*}{$\begin{array}{c}1.0 \\
1.1\end{array}$} & \multicolumn{2}{|c|}{2.0} \\
\hline & & & & $\mathrm{P} 1$ & $\mathrm{P} 2$ \\
\hline 1 & Web & 18 & $\sqrt{ }$ & $\sqrt{ }$ & $\sqrt{ }$ \\
\hline 2 & Escritorio & 8 & $\sqrt{ }$ & $\sqrt{ }$ & \\
\hline 3 & App Móvil & 0 & & & \\
\hline & Total & 26 & & & \\
\hline
\end{tabular}

Fuente: Elaboración propia

Análisis: Se observa que se han recopilado un total de 26 diferentes tipos de ejemplos que demuestran el funcionamiento del estándar WaterML de las cuales 18 son recursos de aplicaciones web que equivale a un $69.23 \%, 8$ son de escritorio (30.77\%) y no se ha encontrado aplicaciones móviles como recurso para el uso de WaterML.

Aunque se han revisado un conjunto diverso de ejemplos, la mayoría de las aplicaciones siguen el siguiente patrón de uso de WaterML (Figura 7):

- La colección de sensores es representada por las posiciones de los lugares de interés en un mapa (Figura 7A).

- Cada posición viene descrita por un conjunto de atributos siendo uno de ellos la serie temporal (Figura 7B).

- Cada sensor individual es un lugar de interés (FeatureOfInterest) generalmente representado por una posición puntual. Además, se añade una lista de valores adquiridos en tiempos determinados. Todo ello se empaqueta en un documento WaterML que puede ser recuperado a partir de una petición SOS (Figura 7C).

- La colección de sensores puede ser recuperada usando una petición a un servicio WFS (Figura 7C). 


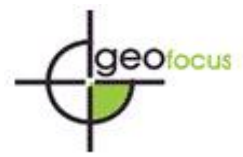

Salas Challco B. M., Masó Pau, J. y Pesquer Mayos, L. (2017): "Estudio del estándar WaterML", GeoFocus (Artículos), $n^{\circ}$ 19, p. 129-150. ISSN: 1578-5157 http://dx.doi.org/10.21138/GF.485

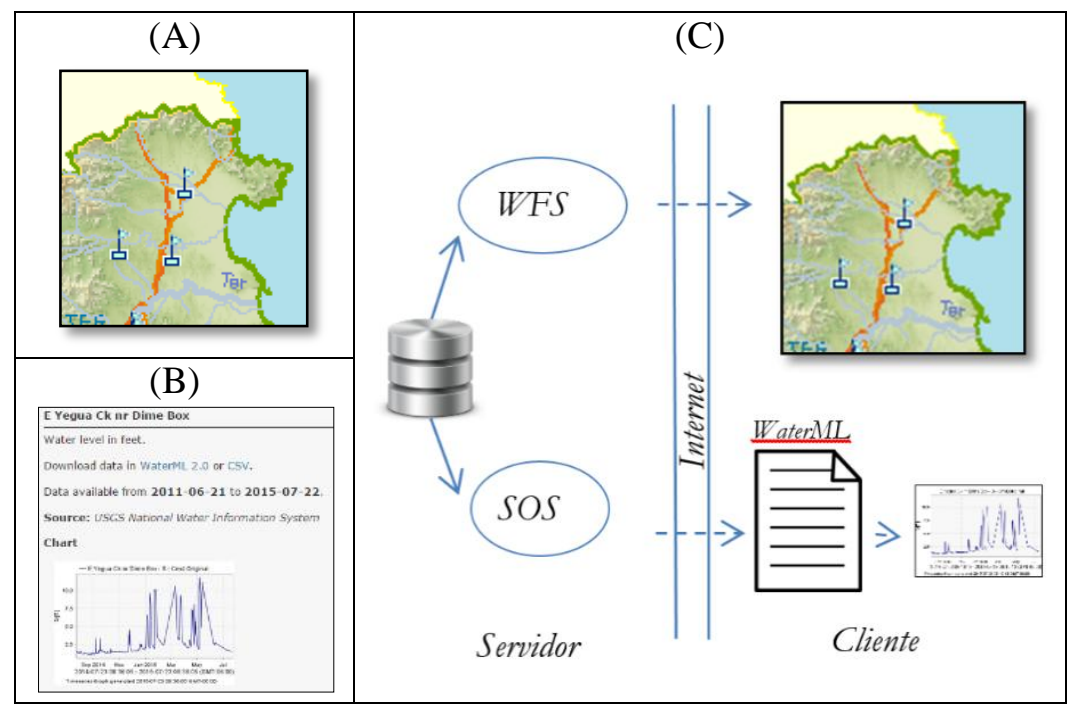

Figura 7: Uso combinado de WaterML y otros estándares (A) Posición de los Lugares de Interés, (B) Timeseries y (C) Modelo Cliente-Servidor.

Fuente: Elaboración propia

\section{Conclusiones}

WaterML 2.0 es una familia de estándares relacionados y complementarios que permite la codificación de los datos de observaciones del agua para ser utilizado en una amplia variedad de escenarios. Por ejemplo: en el monitoreo de datos hidrológicos (aplicaciones en inundabilidad, caudal ecológico, sequía, etc.); en el análisis de series temporales; en publicación en internet mediante servicios (SOS, WFS) o descargas bajo demanda; y en el apoyo a los gestores y soportes de la toma de decisiones.

WaterML dispone de capacidades muy interesantes para algunos metadatos (unidades, magnitudes, calidad, fuente, etc.) a nivel de observación que otros formatos propios de bases SIG sólo disponen a nivel de atributo (p. ej. en un XML con metadatos más generales).

$\mathrm{Su}$ implementación todavía no está muy extendida, algunas implementaciones de visualizadores web confiesan no usar WaterML sino simplificaciones basadas en codificaciones JSON o CSVs, en otros casos el usuario puede descargar los datos en formato WaterML bajo demanda y son muy escasas las implementaciones en servicios interoperables.

No se han encontrado herramientas que puedan usar WaterML como formato de entrada ni para análisis estadísticos ni para alimentar una simulación de un modelo hidrológico. La excepción genérica es Excel que permite visualizar WaterML a partir de su cinta de opciones (importar datos desde la web).

No se ha encontrado ningún cliente que ofrezca la posibilidad de cargar información de un servidor que no sea el suyo propio.

En realidad WaterML 2.0 parte 1, describe básicamente series temporales sin demasiado énfasis en las peculiaridades hidrológicas, a pesar de ello los ejemplos muestran el dominio de aplicación natural. En esta línea, OGC ha creado un grupo de trabajo enfocado a la representación de series temporales TimeseriesML 1.1 (Tomkins, 2014). Este trabajo está motivado según las exigencias en hidrología y meteorología, pero el estándar será diseñado para ser útil en cualquier aplicación que implica el muestreo periódico de información espacial referenciada y no solo para observaciones hidrológicas. 


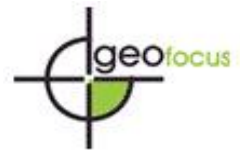

Salas Challco B. M., Masó Pau, J. y Pesquer Mayos, L. (2017): "Estudio del estándar WaterML”, GeoFocus (Artículos), $n^{\circ}$ 19, p. 129-150. ISSN: 1578-5157 http://dx.doi.org/10.21138/GF.485

Finalmente indicar que la forma más útil para suministrar datos hidrológicos es a través de un servicio SOS que permite acceder a las observaciones temporales y de sus localizaciones geográficas de los datos (Feature of Interest), en la Tabla 4 corresponde al registro 18 NRFA /CEH.

\section{Agradecimientos}

Este trabajo se ha realizado gracias a la financiación económica de estudios del Máster en Teledetección y SIG en la Universitat Autònoma de Barcelona de España por la "Beca Presidente de la Republica" del Programa Nacional de Becas y Crédito Educativo (PRONABEC) del Ministerio de Educación de la República del Perú y gracias a los recursos de European Union's Horizon 2020 Programme under Grant Agreement 641821 WaterInnEU.

\section{Referencias bibliográficas}

Almoradie A., Jonoski A., Popescu I., Solomatime D. (2013): "Web Based Access to Water Related Data Using OGC WaterML 2.0", International Journal of Advanced Computer Science and Applications (IJACSA), EnviroGRIDS Special Issue on "Building a Regional Observation System in the Black Sea Catchment", pp. 83-89.

Ames D.P., Horsburgh, J.S., Cao, Y., Kadlec, J., Whiteaker, T., Valentine, D. (2012): "HydroDesktop: Web services-based software for hydrologic data discovery, download, visualization, and analysis". Environmental Modelling \& Software 37, pp.146-156.

Bermudez L. E., Masó J., Capdevila J. (2012): “Open Geospatial Consortium (OGC)” en Bernabé-Poveda M.A., López-Vázquez C.M. (Eds): Fundamentos de las Infraestructuras de Datos Espaciales. Madrid, UPM-Press, Serie Científica. ISBN: 978-84-939196-6-5, pp. 265274.

Botts M., Robin A. (Eds.) (2014): "OGC SensorML: Model and XML Encoding Standard". Open Geospatial Consortium. OGC Encoding Standard. OGC 12-000. Version: 2.0.0. [Consulta: 05-03-2015] Disponible en: http://www.opengeospatial.org/standards/sensorml.

Bröring A., Stasch C., Echterhoff J. (Eds.) (2012): "OGC Sensor Observation Service Interface Standard". Open Geospatial Consortium. OpenGIS Implementation Standard. OGC 12-006. Version: 2.0. [Consulta: 05-03-2015] Disponible en: http://www.opengeospatial.org/standards/sos.

Burillo M., Sanz J. M. (1960): "Manual de Hidrología de España". Madrid, Vinchés, Librero. 267 Págs.

Canchala A. (2015): "UML, ejemplo sencillo sobre modelado de un proyecto. Microsoft. [Consulta: 05-07-2015] Disponible en: https://msdn.microsoft.com/es-es/library/bb972214.aspx.

Car N. J., Moore G. A. (2011): “Towards standardising irrigation DSS inputs data formats through adaptation of the WDTF/WaterML" In Chan, F., Marinova, D. and Anderssen, R.S. (Eds) MODSIM2011, 19th International Congress on Modelling and Simulation. Modelling and Simulation Society of Australia and New Zealand, December 2011, pp. 3286-3292.

Castaño S., Gómez-Alday J. J, Sanz D. (2008): "Teledetección y sistemas de información geográfica en la gestión de las aguas subterráneas" en Barceló D. (Coord): Aguas continentales. Gestión de recursos hídricos, tratamientos y calidad de agua. Madrid, Cyan, Proyectos y Producciones Editoriales, S.A, pp. 55-70. 


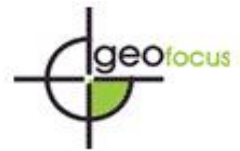

Salas Challco B. M., Masó Pau, J. y Pesquer Mayos, L. (2017): "Estudio del estándar WaterML”, GeoFocus (Artículos), $n^{o}$ 19, p. 129-150. ISSN: 1578-5157 http://dx.doi.org/10.21138/GF.485

Cox S. (Ed) (2011): “Observations and Measurements - XML Implementation". Open Geospatial Consortium. OGC Implementation. OGC 10-025r1. Version: 2.0. [Consulta: 05-032015] Disponible en: http://www.opengeospatial.org/standards/om.

Cox S. (Ed) (2013): "OGC Abstract Specification - Geographic information - Observations and measurements". OGC and ISO 19156:2011(E). OGC Standard: Abstract Specification. OGC 10-004r3. Version: 2.0. [Consulta: 05-03-2015] Disponible en: http://www.opengeospatial.org/standards/om.

Diez Hochleitner, R. (1995): "El agua en el mundo", El campo. Servicio de Estudios BBV del Banco Bilbao Vizcaya (BBV) 132, pp. 7-14.

Geoconnexion (2013): "New Steps Toward Sharing Hydrological Data". OGC World. Geoconnexion International Magazine, pp. 25-26. [Consulta: 09-06-2015] Disponible en: $\mathrm{http} / / / \mathrm{www}$.geoconnexion.com/articles/new-steps-toward-sharing-hydrological-data/.

Giuliani G., Rahman K., Ray N., Lehmann A. (2013): “OWS4SWAT: Publishing and Sharing SWAT Outputs with OGC standards", International Journal of Advanced Computer Science and Applications (IJACSA), EnviroGRIDS Special Issue on "Building a Regional Observation System in the Black Sea Catchment, pp. 90-98.

Gutiérrez A., Martínez R. (2001): “XML a través de ejemplos”. Ra-Ma. Madrid. 490 Págs.

Hedler M., Montero M., Kutscherauer N. (2011): "Schematron: Effiziente Business Rules für XML-Dokumente". Heidelberg. Dpunkt Verlag. 152 Págs.

Hussain A., Wu W., Anzaldi G., Abecker A. (2015): "Implementation of OGC Compliant Framework for Data Integration in Water Distribution System", 13th Computer Control for Water Industry Conference, CCWI 2015. Procedia Engineering 119, pp. 1366-1374.

ISO/IEC 19757-3 (2006): "ISO/IEC 19757-3:2006 Information technology -- Document Schema Definition Language (DSDL) -Part 3: Rule-based validation -Schematron". ISO/IEC 19757-3:2006(E). [Consulta: 14-07-2015] Disponible en: http://www.schematron.com/.

Jackson S. R. (2014): "RiverML: A Harmonized Transfer Language for River Hydraulic Models". Thesis Presented to the Faculty of the Graduate School of The University of Texas at Austin in Partial Fulfillment of the Requirements for the Degree of Master of Science in Engineering The University of Texas at Austin August, 2014. [Consulta: 13-10-2015] Disponible en: https://repositories.lib.utexas.edu/bitstream/handle/2152/26181/JACKSONTHESIS-2014.pdf?sequence $=1$.

Kadlec J., StClair B., Ames D. P., Gill R. A. (2015): "WaterML R package for managing ecological experiment data on a CUAHSI HydroServer", Ecological Informatics 28, pp19-28.

Khattar R. M., Ames D. (2014): "OGC and HIS: Implementing WFS and WaterML2 for HydroServer" Proceedings on 7th International Congress on Environmental Modelling and Software, San Diego, California, USA, D.P. Ames, N. Quinn (Eds.)

Lefort L. (2009): Welcome to the hydrology domain working group. OGC Making location count. [Consulta: 02-07-2015] Disponible en: http://external.opengeospatial.org/twiki_public/HydrologyDWG/WebHome.

Llamas J. (1993): "Hidrología General. Principios y aplicaciones". Bilbao. Universidad del País Vasco.

McEnery J. A., McKee P. W., Shelton P., Ramsey W. (2013): "Hydrologic information server for benchmark precipitation dataset", Computers \& Geosciences 50, pp. 145-153. 


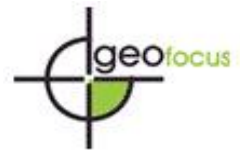

Salas Challco B. M., Masó Pau, J. y Pesquer Mayos, L. (2017): "Estudio del estándar WaterML", GeoFocus (Artículos), $n^{\circ}$ 19, p. 129-150. ISSN: 1578-5157 http://dx.doi.org/10.21138/GF.485

Open Geospatial Consortium (2015): "OGC Standards And Supporting Documents". OGC. [Consulta: 20-03-2015] Disponible en: http://www.opengeospatial.org/standards.

Portele C. (Ed.) (2007): “Opengis Geography Markup Language (GML) Encoding Standard”. Open Geospatial Consortium Inc. OGC 07-036. Version: 3.2.1. [Consulta: 05-03-2015] Disponible en: http://www.opengeospatial.org/standards/gml.

Portele C. (Ed.) (2012): “OGC Geography Markup Language (GML) — Extended schemas and encoding rules". Open Geospatial Consortium. OpenGIS Implementation Standard. OGC 10129r1. Version: 3.3.0. [Consulta: 05-03-2015] Disponible en: http://www.opengeospatial.org/standards/gml.

Serra I. (2015): "Notas de Prensa del OGC". OGC. [Consulta: 20-06-2015] Disponible en: http://external.opengeospatial.org/twiki_public/ILAFpublic/NoticiasOGC\#A_10_4704_4715_ WaterML_2.0_Part_2:_Ratings_44_Gaugings_and_Sections_disponible_para_comentarios.

Sheahan P., Taylor P. (2014): "WaterML 2.0 Standards Working Group Charter". Open Geospatial Consortium. [Consulta: 11-06-2015] Disponible en: http://www.opengeospatial.org/projects/groups/waterml2.0swg.

Sijbertsma J., Hodge P. (2013): "WaterML2.0 and ESRI". Palmerston North. Horizons Regional Council, Palmerston North. New Zealand Esri User Conference 2013. [Consulta: 09-06-2015] Disponible en: http://www.eagle.co.nz/nzeuc/graphics/etemp/Jorn\%20Sijbertsma_WaterML2.pdf.

Stevens P., Pooley R. (2002): "Utilización de UML en Ingeniería del Software con objetos y componentes." Madrid. Pearson Educación, S.A. Primera Edición.

Taylor P. (Ed.) (2010): "OGC Harmonising standards for water observation data - Discussion Paper". Open Geospatial Consortium Inc. OGC 09-124r2. [Consulta: 05-03-2015] Disponible en: https://portal.opengeospatial.org/files/?artifact_id=39090.

Taylor P. (Ed.) (2014) "OGC WaterML 2.0: Part 1 - Timeseries". OGC Implementation Standard - Corrigendum. OGC 10-126r4. Version: 2.0.1. [Consulta: 05-03-2015] Disponible en: http://www.opengeospatial.org/standards/waterml.

Taylor P. (Ed.) (2015) "WaterML2.0: part 2 - Ratings, Gaugings and Sections". OGC Draft Implementation Specification. OGC 15-018. Version: 0.0.3. [Consulta: 03-06-2015] Disponible en: http://www.opengeospatial.org/standards/waterml.

Tomkins J. (Ed.) (2014): “TimeSeriesML 1.0 Standards Working Group Charter". Open Geospatial Consortium. SWG Charter. OGC 14-092r4. [Consulta: 05-06-2015] Disponible en: https://portal.opengeospatial.org/files/60856.

UNESCO (2015): "El agua dulce". United Nations Educational, Scientific and Cultural Organization. [Consulta: 15-07-2015] Disponible en: http://www.unesco.org/new/es/naturalsciences/environment/water/ .

Vaccari L., Craglia M., Borzacchiello M. T. (2013): "D3.1 Multidisciplinary Interoperability Requirements specification”. GEOWOW. GEOWOW-WP3-DEL-D3 1-v0 52. [Consulta: 1106-2015] Disponible en: http://www.geowow.eu/downloads/GEOWOW-WP3-DEL-D3.1v1.0.pdf.

Vretanos P.A. (Ed.) (2010): "OpenGIS Web Feature Service 2.0 Interface Standard (also ISO 19142)". OGC 09-025r1 and ISO/DIS 19142. Version: 2.0.0. [Consulta: Disponible en: http://www.opengeospatial.org/standards/wfs. 


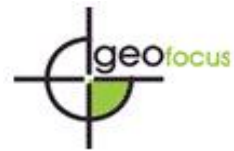

Salas Challco B. M., Masó Pau, J. y Pesquer Mayos, L. (2017): "Estudio del estándar WaterML”, GeoFocus (Artículos), $n^{\circ}$ 19, p. 129-150. ISSN: 1578-5157 http://dx.doi.org/10.21138/GF.485

W3C España (2015): "Guía Breve de Tecnologías XML”. España. World Wide Web Consortium (W3C). [Consulta: 01-07-2015] Disponible en: http://www.w3c.es/Divulgacion/GuiasBreves/TecnologiasXML.

Walker, G., Taylor P., Cox S., Sheahan P. (2009): "Water Data Transfer Format (WDTF): Guiding principles, technical challenges and the future”. In Anderssen, R.S., R.D. Braddock and L.T.H. Newham (eds) 18th World IMACS Congress and MODSIM09 International Congress on Modelling and Simulation. Modelling and Simulation Society of Australia and New Zealand and International Association for Mathematics and Computers in Simulation, July 2009, pp. 4381-4387.

World Metereorological Organization (2015): "Homs- Sistema De Hidrología Operativa para Fines Múltiples". Geneva. WMO. [Consulta: 14-07-2015] Disponible en: http://www.wmo.int/pages/prog/hwrp/homs/homs_es.html.

Yu J., Taylor P., Cox S. J. D., Walker G. (2015): "Validating observationdatainWaterML2.0", Computers \& Geosciences 82, pp. 98-110.

Yu J., Taylor P., Cox S., Walker G. (2011): "Validating water resources described in WaterML 2.0", EGU General Assembly 2011, Geophysical Research Abstracts Vol. 13, EGU2011-9649. [Consulta: 12-09-2015] Disponible en: http://meetingorganizer.copernicus.org/EGU2011/EGU2011-9649.pdf.

Yu J., Taylor P., Cox S., Walker G. (2012): "Towards validating observation data in WaterML 2.0", 10th International Conference on Hydroinformatics HIC 2012, Hamburg, Germany. [Consulta: 12-09-2015] Disponible en: https://wiki.csiro.au/download/attachments/85753968/HYA00320-00598-WaterML2-

Validation_final.pdf?version=1\&modificationDate=1380678623767\&api=v2 .

Zaslavsky I., Valentine D., Whiteaker T. (Eds.) (2007): "CUAHSI WaterML". OGC. Discussion Paper. OGC 07-041r1. Version 0.3.0. [Consulta: 04-03-2015] Disponible en: http://portal.opengeospatial.org/files/?artifact_id=21743. 